\title{
Lu-Hf Isotopic Data of the Mbé-Sassa-Mbersi Tonalite (Central Cameroon Domain): Indicator of ca. 1.0 Ga Juvenile Tonian Magmatism in the Region
}

\author{
Alliance Nicaise Saha-Fouotsa ${ }^{*}$, Rigobert Tchameni ${ }^{2}$, Olivier Vanderhaeghe ${ }^{3}$, \\ Armin Zeh4, Periclex Martial Fosso Tchunte2 ${ }^{2}$ Aurélien Eglinger ${ }^{5}$, Emmanuel Negue Nomo ${ }^{6}$, \\ Pierre Barbey ${ }^{7}$
}

${ }^{1}$ Department of Earth Sciences, Faculty of Science, University of Maroua, Maroua, Cameroon

${ }^{2}$ Department of Earth Sciences, Faculty of Science, University of Ngaoundéré, Ngaoundéré, Cameroon

${ }^{3}$ GET, Université de Toulouse, UPS, CNRS, IRD, CNES, Toulouse, France

${ }^{4}$ KIT-Karlsruher Institut für Technologie, Campus Süd, Institut für Angewandte Geowissenschaften, Abteilung Mineralogie und Petrologie, Karlsruhe, Germany

${ }^{5}$ Université de Lorraine, CNRS, GeoRessources, Nancy, France

${ }^{6}$ Department of Earth Sciences, Faculty of Science, University of Yaoundé I, Yaoundé, Cameroon

${ }^{7}$ Université de Lorraine, CNRS, CRPG, Nancy, France

Email: nicsaha@yahoo.fr

How to cite this paper: Saha-Fouotsa, A N., Tchameni, R., Vanderhaeghe, O., Zeh, A., Tchunte, P. M. F., Eglinger, A., Nomo, E. N., \& Barbey, P. (2021). Lu-Hf Isotopic Data of the Mbé-Sassa-Mbersi Tonalite (Central Cameroon Domain): Indicator of ca. 1.0 Ga Juvenile Tonian Magmatism in the Region. Journal of Geoscience and Environment Protection, 9, 1-19. https://doi.org/10.4236/gep.2021.99001

Received: August 2, 2021

Accepted: September 3, 2021

Published: September 6, 2021

\begin{abstract}
The Pan-African/Brasiliano orogenic belts are a part of numerous Neoproterozoic orogenic belts that belong to the long-lived orgenic cycle that distancing phase started at the Tonian around $1.0 \mathrm{Ga}$. The Tonian magmatism fairly documented in the Neoproterozoic belts of Borborema Province (NE-Brazil), seemed so far inexistent in the Central African Orogenic Belt (CAOB) although these two belts were geologically correlated. Through the $\mathrm{Lu}-\mathrm{Hf}$ geochronological analysis on zircon of tonalite, the present work, coupled with the previous data, suggests the existence of a Tonian age magmatism in the Central Cameroon Domain of the CAOB although the latter is much reworked. The Nguesseck tonalite outcrops in the northern part of the Mbé-Sassa-Mbersi region, in the northern edge of Central Cameroon Domain of the CAOB and in the Tcholliré-Banyo shear zone (TBSZ). The Lu-Hf data obtain on the zircon grains of this tonalite reveal juvenile $\mathrm{Hf}_{\mathrm{TDM}}$ age of ca. 1.0 Ga. This age, combined to the previous geochemical data suggests that the protholites of this tonalite would have extracted from the source during the distancing phase (rifting and oceanization) of the Pan-african/Brasiliano orogeny at the early Neoproterozoic.
\end{abstract}


Copyright $\odot 2021$ by author(s) and Scientific Research Publishing Inc. This work is licensed under the Creative Commons Attribution International License (CC BY 4.0).

http://creativecommons.org/licenses/by/4.0/

\section{Keywords}

Mbé-Sassa-Mbersi, Central Cameroon Domain, Central African Orogenic

Belt, Tonian magmatism, Lu-Hf Isotopic Data

\section{Introduction}

The central Cameroon Domain is the part of Central African Orogenic Belt which is correlated with the Pan-African/Brasiliano orogenic belts of the Borborema Province in NE-Brazil (Figure 1; Neves, 2003; Guimarães et al., 2012). The Proterozoic evolution in the Boeborema Province would have started from the assembly of a supercontinent named Atlantica (Rogers, 1996) until its fragmentation during the late Mesoproterozoic to early Neoproterozoic (Neves, 2003; Van Schmus et al., 2008; Guimarães et al., 2012). There were initially several failed attempts of this fragmentation towards the end of Paleoproterozoic $(\sim 1.8-1.7 \mathrm{Ga})$ and the beginning of Mesoproterozoic $(\sim 1.52 \mathrm{Ga})$ before intensification in the beginning of Neoproterozoic ca. $1 \mathrm{Ga}$ (Souza \& Moura 1995; Van Schmus et al., 1999; Kozuch, 2003; Santos et al., 2010; Guimarães et al., 2012). The Cariris Velhos belt is a part of the Borborema Province which exhibits a petrographic complex dated at the Tonian (1.0 - $0.85 \mathrm{Ga}$ ) (Santos, 1995; Brito Neves et al., 2001; Tack et al., 2001; Kozuch, 2003; Santos et al., 2010; Guimarães et al., 2012). In spite of the similarity between the Borborema Province (NE-Brazil) and Central African Orogenic Belt (Van Schmus et al., 2008), the Tonian age rocks presented in the Cariris Velhos suite have not yet been described in the Cameroon part of Central African Orogenic Belt, though the biotite granite of the Ouaddaï (Chad) yield concordant inherited zircon cores crystallised around 1.05 Ga (Djerossem et al., 2020). These rocks were already described in the West Congo Belt (Araçuai Orogen) where thick rift-related alkaline volcanic-sedimentary basin and anorogenic granites of 1000 - $910 \mathrm{Ma}$ are exposed (Tack et al., 2001; Van Schmus et al., 2008; Guimarães et al., 2012). However, ca. 1.0 Ga dolerite dykes with continental tholeiite composition outcropped in the Paleoproterozoic formation and $1167 \pm 61$ Ma granite (Yobé) in far south-east of Cameroon and South-west of Central African Republic. These dolerites are assigned to the pre-Panafrican extension phase (Vicat \& Pouclet 1995; Vicat et al., 1997, 2001). This paper presents Lu-Hf isotopic data on zircon from the Nguessek Tonalite near the Tcholliré-Banyo shear zone in the Mbé-Sassa-Mbersi region. These data provide additional information concerning the evolution of the early Neoproterozoic in the central Cameroonian domain of the Central Africa orogenic belt.

\section{General Geological Context}

The similarities in geology, gold mineralization and old fluviodeltaic deposits in the São-Francisco/Congo, Amazonian, West African and Rio de la Plata cratons 


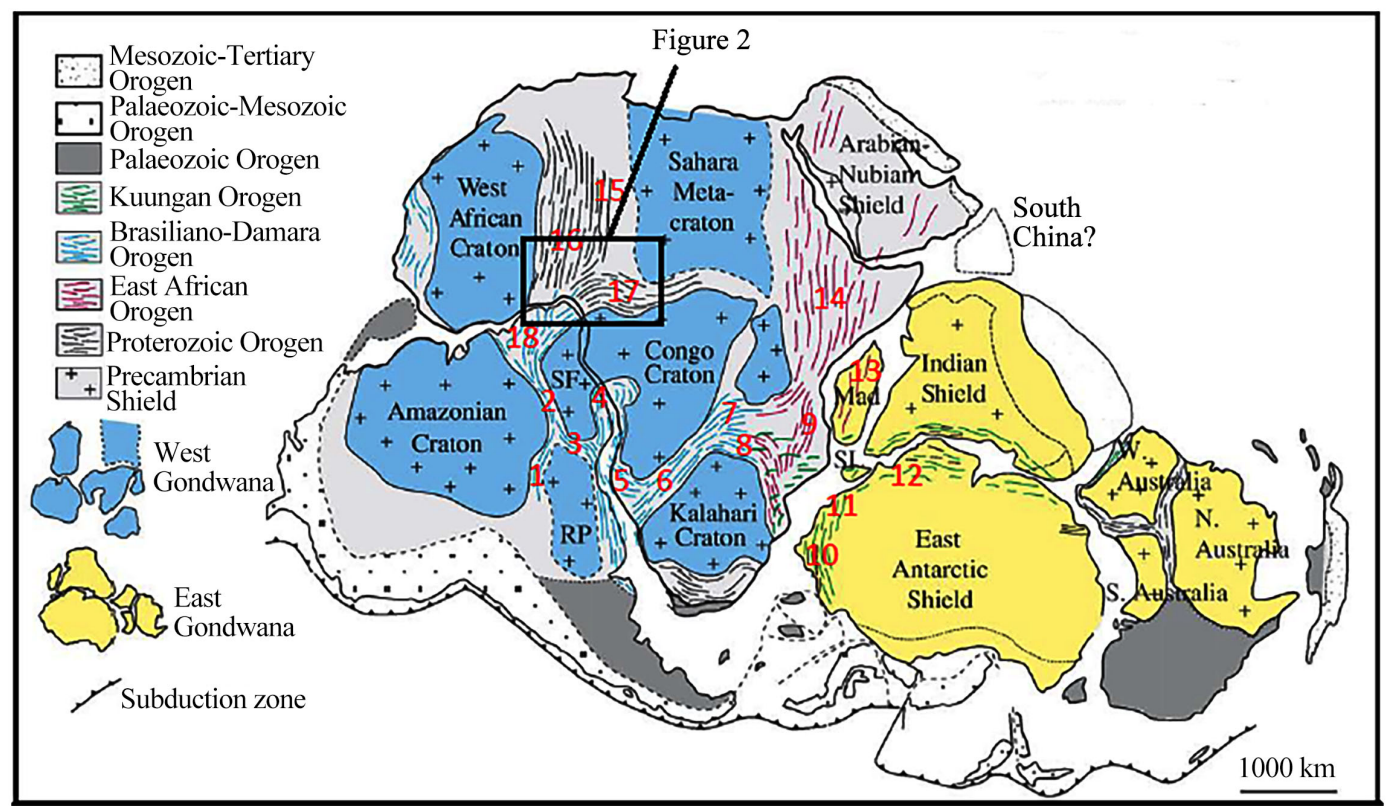

Figure 1. Gondwana Supercontinent (modifyed after Gray et al., 2008). Numbers 1 to 18 represent some Pan-African belt. 1: Paraguai (P); 2: Araguaia (Ag); 3: Brasiliano (Br); 4: Araçuai (Ac); 5: Kaoko (Ka); 6: Damara (Da); 7: Lufilien arc (La); 8: Katanga/Zambèze (K/Z); 9: Mozambicaine (Mo); 10: Sør Rondane Massifs (SR); 11: Yamato Mountain (Y); 12: Lützow-Holm bay (LF); 13: Madagascard (Mad); 14: Tanzanianne (Ta); 15: Hoggar (Ho); 16: Transahariennes/Dahomeides ( $\mathrm{Tr} / \mathrm{Dh})$; 17: Centrale African Orogenic Belt (CAOB); 18: Borborema (Bo).

allowed some authors to suggest that, these cratons formed a same continent named Atlantica, at the end of the Transamazonian/Eburnean orogeny (Ledru et al., 1994; Bossière et al., 1996; Rogers, 1996; Teixeira et al., 1999; Norcross et al., 2000; Neves, 2003). Paleomagnetic poles for mafic rocks in Amazonia allowed to suggest that Atlantica was the part of Rodinia supercontinent at the late Mesoproterozoic (Tohver et al., 2002). Atlantica would be the basement of the Pan-African Borborema, Araguia, Nigeria and Cameroon orogenic domains (Neves, 2003; Guimarães et al., 2012). Excluding continental rifting stages between 1.8 and $1.5 \mathrm{Ga}$ mark by ubiquitous taphrogenesis, this continent remained unaffected for about $1 \mathrm{Ga}$ (Neves, 2003). But, around $1 \mathrm{Ga}$ at the early Neoproterozoic (Tonian), this portion of the crust is witness of an important magmatic event in Borborema province and Araguaia Group correlates with important rifting episodes in central-western Africa and anorogenic magmatism in the São Francisco, Congo, and Amazonian cratons (Vicat \& Pouclet 1995; Souza \& Moura 1995; Vicat et al., 1997, 2001; Neves, 2003; Guimarães et al., 2012; Viana \& Battilani 2014).

The various studies carried out show that Tonian magmatism takes place between 1000 and $850 \mathrm{Ma}$ in Brazil and Africa (Santos, 1995; Vicat \& Pouclet 1995; Vicat et al., 1997, 2001; Van Schmus et al., 1999, 2008; Da Silva Filho et al., 2000; Brito Neves et al., 2001; Tack et al., 2001; Kozuch, 2003; Neves, 2003; Santos et al., 2010; Guimarães et al., 2012). This magmatism which is materialized in the outcrops by volcano-sedimentary formations associated with meta-mafic and 
felsic volcanic and plutonic rocks, orthogneisses and dolerite dykes show continental tholeiite and alkaline signature (Souza \& Moura 1995; Vicat et al., 2001; Neves, 2003; Guimarães et al., 2012; Viana \& Battilani 2014). In the Central Cameroon Domain in Central African Orogenic Belt (Figure 2(a) and Figure 2(b)), the tonian ages are mentioned in the biotite-muscovite granitoids ( 926 $\mathrm{Ma})$ and amphibole-biotite gneiss ( $\sim 889 \mathrm{Ma})$ respectively in the Ngaoundere cliff and Meiganga area (Tchameni et al., 2006; Ganwa et al., 2011). But these ages have been interpreted as inherited and of no geological significance, probably due to the lack of more detailed studies.

The Central Cameroon Domain is characterised by the emplacement of magmatic rocks during the Pan-African orogeny that display a variety of petrological and geochemical signatures (Figure 2(b)). Plutonic rocks consist of diorite, tonalite, trondhjemite, granodiorite and granite (Toteu et al., 2001, 2004; Njanko et al., 2006; Tchameni et al., 2006; Ganwa et al., 2008; Naïmou et al., 2014; Saha-Fouotsa 2018; Saha-Fouotsa et al., 2018, 2019). They have been dated from ca. 650 to $570 \mathrm{Ma}$ and their calc-alkaline signatures, to high-K calc-alkaline for the youngest ones, have been attributed to mixing between a juvenile source and magmas issued from partial melting of Archean to Paleoproterozoic crust (Tchameni et al., 2006; Kwékam et al., 2010; Mbassa et al., 2016; Tchakounté et al., 2017; Saha-Fouotsa et al., 2019). With regard to the juvenile source, two
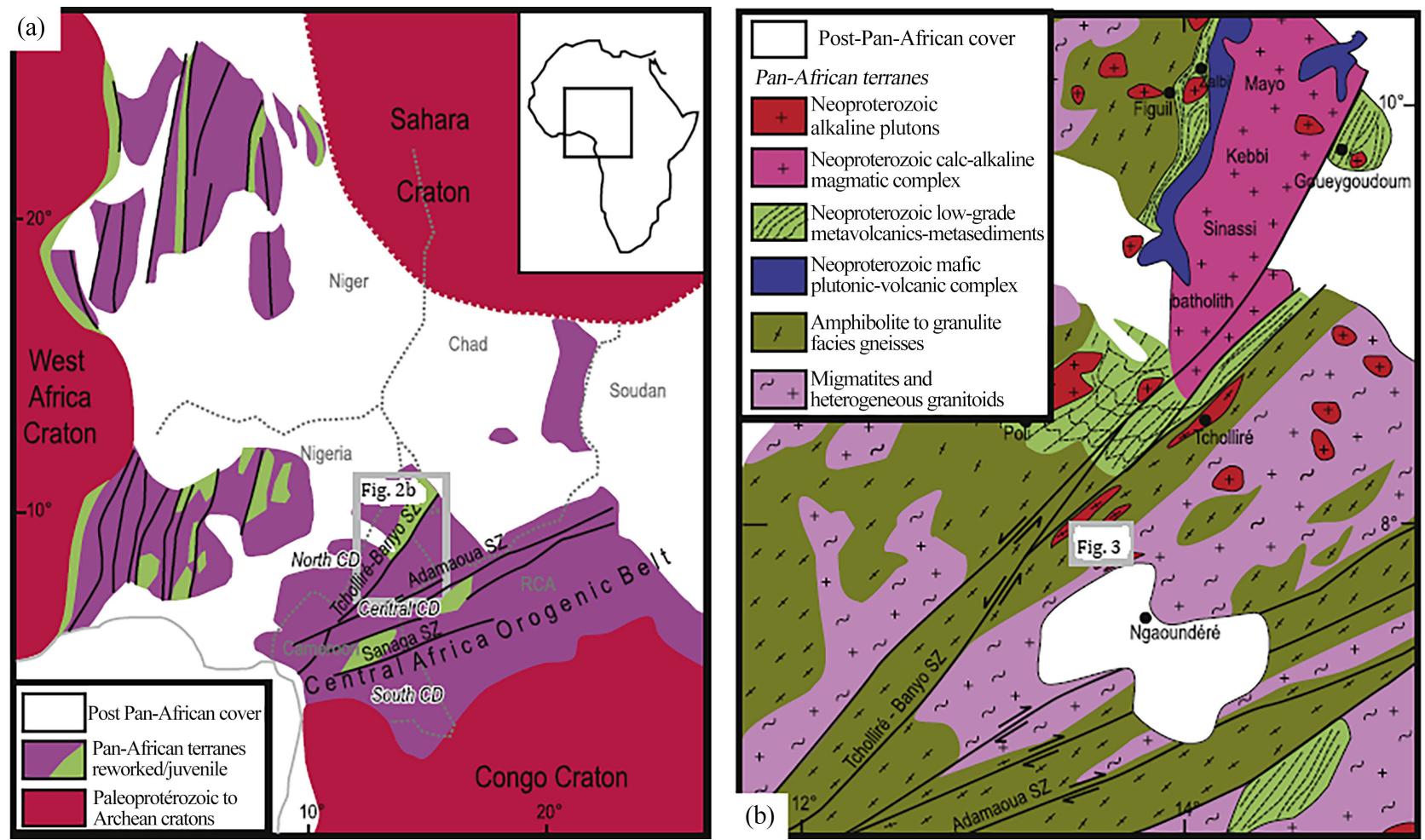

Figure 2. (a) Tectonic map of Western Gondwana showing the position of the Central African Orogenic Belt pinched in between the West-African craton, the Congo craton and the Sahara metacraton (modified after Küster and Liégeois, 2001; Toteu et al., 2004; Saha-Fouotsa et al., 2019). The position of the map at the scale of Africa is shown in the inset. (b) Geology of the Central African Orogenic in Cameroon and southern Chad (modified after Toteu et al., 2001, 2004; Pinna et al., 1994; Saha-Fouotsa et al., 2019). The location of the map is shown by a grey rectangle on Figure 2(a). Abbreviation: CD = Central Domain; SZ = Shear Zone. 
propositions have been made: 1) an enriched mantle or partial melting triggered by post-collisional delamination of the subcontinental lithospheric mantle (Tchameni et al., 2006; Kwékam et al., 2010); and 2) partial melting of Neoproterozoic oceanic crust tectonically accreted with the continental terranes (Mbassa et al., 2016; Saha-Fouotsa 2018; Saha-Fouotsa et al., 2019).

In this paper, we bring new isotopic data which allows to reconsider the interpretation about tonian ages in Cameroon and to discuss the source of the juvenile magmatism at Neoproterozoic in order to improve the reconstruction of the tectono-magmatic history of the Mbé-Sassa-Mbersi region and therefore that of the Central Cameroon Domain.

\section{Geological Background of Studied Rocks}

The Mbé-Sassa-Mbersi region is situated at the northern edge of the Central Cameroon domain, along the Tcholliré-Banyo shear zone (TBSZ) between the Tcholliré and Banyo towns, about $60 \mathrm{~km}$ north of the Ngaoundéré city (Figure 3). Saha-Fouotsa (2018) and Saha-Fouotsa et al. $(2018,2019)$ identify in this region, a diversity of lithological formations including: 1) mafic rocks in which

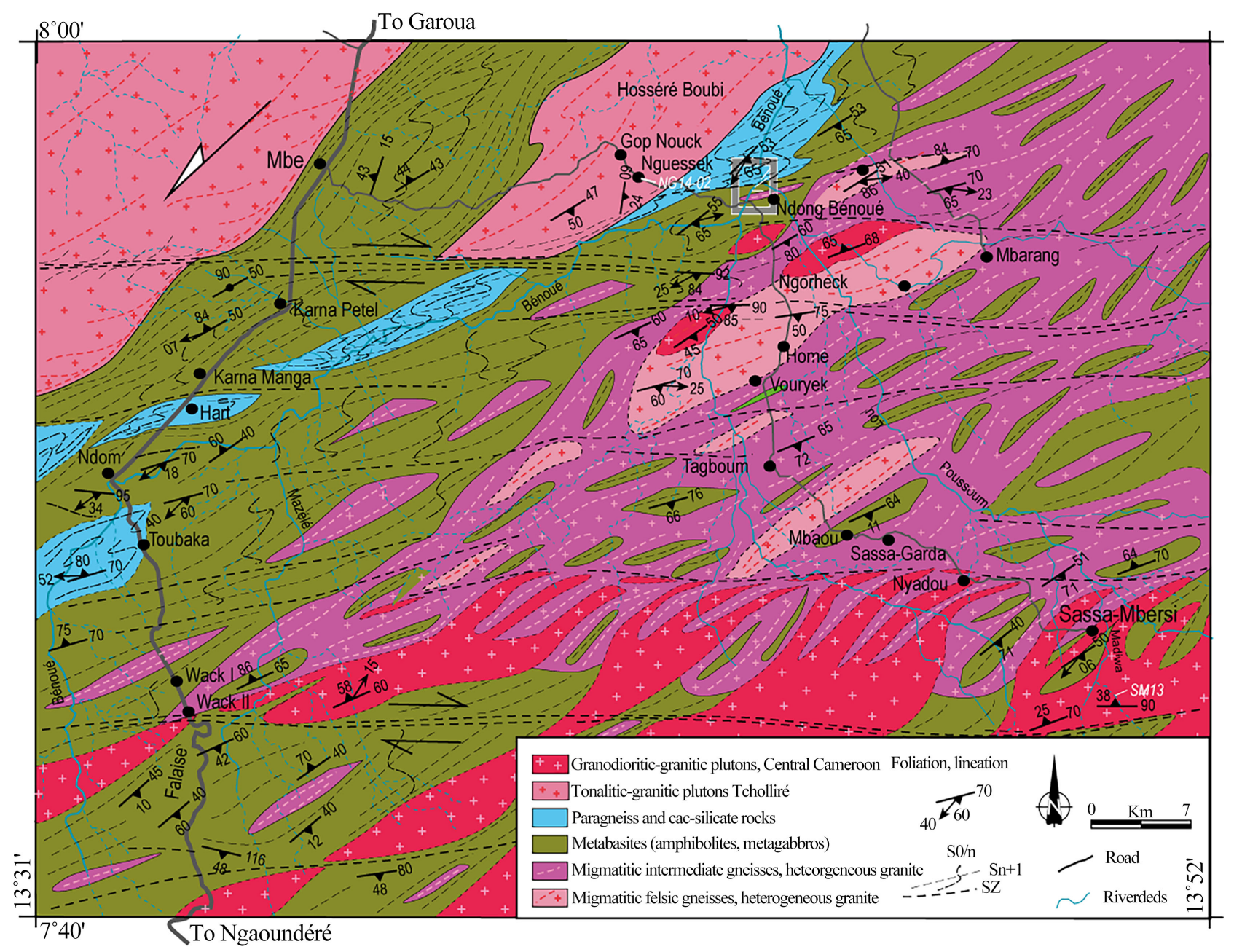

Figure 3. Geological map of the Mbé-Sassa-Mbersi area. 
some present concordant $\mathrm{U}-\mathrm{Pb}$ ages of Pan-African.; 2) paragneisses displaying ages with Archean, Paleoproterozoic and Neoproterozoic sources and calc-silicate gneiss; 3) heterogeneous, migmatitic gneisses with an intermediate to felsic composition, and 4) monzodioritic to granitic plutons and dikes more or less affected by solid-state deformation, showing I-type chemical signature with three series which are tonalites-trondhjemites with a magnesian medium-K signature, hornblende-biotite granites with a magnesian high-K signature and biotite-granite with a ferroan shoshonitic signature. All these formations are affected by ductile polyphase deformation marked by tectonic nappe verging toward SSE (D1), simple shear dominated transpression associated to the Tcholliré-Banyo shear zone evolution (D2) and strain partitioning induced by transpression and links to the Central Cameroon shear zone evolution (D3) (Saha-Fouotsa et al., 2018). The minimum age obtained on metamorphic rims of paragneiss constrain the development of ductile fabric occurring from around $582 \pm 4 \mathrm{Ma}$ under granulite to amphibolite facies metamorphic conditions as constrained by a peak at ca. $1.3 \mathrm{GPa}$ and ca. $770^{\circ} \mathrm{C}$ (Saha-Fouotsa et al., 2019).

The tonalite analysed here, outcrops in the Nguessek locality in the North of the Mbé-Sassa-Mbersi region. The rock is deformed showing alternating quartz-feldspar and amphibole rich layers and preferred orientation of grains (Saha-Fouotsa et al., 2018). The deformation of this tonalite is described by Saha-Fouotsa et al. (2018) as belonging to the first deformation phase in the Mbé-Sassa-Mbersi region, which is linked to tectonic nappe verging toward SSE. It showing magnesian medium-K and I-type chemical signature and it emplacement period is constrained by zircon $\mathrm{U}-\mathrm{Pb}$ data at $651 \pm 3 \mathrm{Ma}$ (Saha-Fouotsa et al., 2019). The Rare Earth Elements (REE) patterns fractionation with low heavy REE concentrations, has been interpret by Saha-Fouotsa et al., (2019) as a possible origin by partial melting of mafic material involving fractionation of amphibole and garnet. However, the absence of additional isotopic data did not allow further interpretation of the source of this tonalite. For this paper, we use Lu-Hf isotopic data obtained on the same analysis points of sample of tonalite used for $\mathrm{U}-\mathrm{Pb}$ isotopic data on zircon to improve the interpretation of the source of this rock.

\section{Methodology}

\subsection{Sampling}

Classical field methods were used in this study. Three samples were collected according to their distance on the field and apparent freshness, but only one has been geochronologically analyzed because of their petrographic similarity, both macroscopic and microscopic.

\subsection{Lu-Hf Analytical Method}

Rock sample was crushed with a big and a small jaw crusher at Géosciences Environnement Toulouse (GET) laboratory (France). Then, samples were sieved 
and washed, and the $50 \mu \mathrm{m}$ to $500 \mu \mathrm{m}$ fraction was retained. Zircon grains were concentrated by 1) using heavy liquids (bromoform: density $=2.84 \mathrm{~g} / \mathrm{cm}^{3}$, and diiodomethane: density $=3.31 \mathrm{~g} / \mathrm{cm}^{3}$ ), and 2) magnetic separation with a Frantz isodynamic separator. Subsequently the zircon grains were hand-picked under a binocular microscope, mounted in epoxy blocks, and polished to expose their centre parts. Internal textures and zoning were imaged using combined secondary electron microscopy (SEM), cathodoluminescence (CL) and back-scattered electron (BSE) imaging techniques obtained with a JEOL JSM-6490 instrument coupled with a Gatan MiniCL at the Goethe-Universität Frankfurt am Main (Germany). Subsequently, $\mathrm{U}-\mathrm{Pb}$ and $\mathrm{Lu}-\mathrm{Hf}$ isotopes analyses of zircon grains were carried out successively by laser ablation-inductively coupled plasma mass spectrometry (LA-ICP-MS), using an Thermo-Finnigan ELEMENT and NEPTUNE mass spectrometer coupled to an RESONETIC $193 \mathrm{~nm}$ Excimer laser, all at the Goethe-Universität Frankfurt am Main, Campus Riedberg (Germany). Methods and instruments were the same as described in detail by Gerdes and Zeh (2006, 2009), with modifications explained in Zeh and Gerdes (2012). Laser spots for Lu-Hf (squared spots with an edge length of $40 \mu \mathrm{m}$ ) were placed mostly directly "on-top" of the U-Pb laser spots (from 20 to $30 \mu \mathrm{m}$ in diameter with a typical penetration depth of $\sim 15-20 \mu \mathrm{m}$ ), or within the same zone characterized by CL imaging. Calculations are shown in Table 1.

Table 1. LA-MC-ICPMS Lu-Hf isotope data of zircon from Tonalite sample ofthe Mbé-Sassa-Mbersi region.

\begin{tabular}{|c|c|c|c|c|c|c|c|c|c|c|c|c|c|c|c|c|c|}
\hline $\begin{array}{l}\text { grain/ } \\
\text { domain }\end{array}$ & comm & $\mathrm{bb} /{ }^{173}$ & & $\mathrm{Lu} /{ }^{177} \mathrm{I}$ & $\pm 2 \sigma$ & ${ }^{178} \mathrm{Hf} /{ }^{177} \mathrm{Hf}$ & $\begin{array}{c}{ }^{180} \mathrm{Hf} /{ }^{177} \mathrm{H} \\
\mathrm{f}\end{array}$ & $\begin{array}{l}\operatorname{Sig}_{H f}^{b} \\
(V)\end{array}$ & ${ }^{6} \mathrm{Hf} /{ }^{177} \mathrm{Hf}$ & $2 \sigma^{c}$ & $\begin{array}{c}{ }^{176} \mathrm{Hf} / \\
{ }^{177} \mathrm{Hf}_{(\mathrm{t})}{ }^{\mathrm{d}}\end{array}$ & $\varepsilon H f_{(t)}{ }^{d}$ & $\pm 2 \sigma^{c}$ & $\begin{array}{l}\mathrm{T}_{\mathrm{DM} 2}{ }^{\mathrm{e}} \\
(\mathrm{Ga})\end{array}$ & $\begin{array}{l}\operatorname{age}^{f} \\
(\mathrm{Ma})\end{array}$ & $\pm 2 \sigma$ & conc..$^{g}$ \\
\hline \multicolumn{18}{|c|}{ Sample 1, tonalite (NG14-02) } \\
\hline zA8 & $\mathrm{m}$ & 0.0098 & 0 & 0.00035 & 0 & 1.46724 & 1.88692 & 16 & 0.282626 & 30 & 0.282622 & 8.7 & 1.1 & 1.03 & 650 & 10 & 98 \\
\hline zA9 & $\mathrm{m}$ & 0.0141 & 4 & 0.00049 & 1 & 1.46723 & 1.88687 & 15 & 0.282624 & 28 & 0.282618 & 8.7 & 1.0 & 1.03 & 655 & 10 & 101 \\
\hline zA10 & $\mathrm{m}$ & 0.0094 & 0 & 0.00035 & 0 & 1.46730 & 1.88683 & 19 & 0.282619 & 28 & 0.282615 & 7.7 & 1.0 & 1.06 & 616 & 9 & 99 \\
\hline zA11 & $\mathrm{m}$ & 0.0158 & 3 & 0.00059 & 1 & 1.46726 & 1.88700 & 15 & 0.282606 & 27 & 0.282599 & 7.8 & 0.9 & 1.08 & 645 & 9 & 95 \\
\hline zA12 & $\mathrm{m}$ & 0.0083 & 1 & 0.00031 & 0 & 1.46726 & 1.88695 & 17 & 0.282618 & 27 & 0.282614 & 8.7 & 0.9 & 1.04 & 657 & 10 & 100 \\
\hline zA13 & $\mathrm{m}$ & 0.0074 & 1 & 0.00027 & 0 & 1.46722 & 1.88674 & 16 & 0.282595 & 27 & 0.282592 & 7.6 & 1.0 & 1.09 & 646 & 10 & 86 \\
\hline zA14 & $\mathrm{m}$ & 0.0079 & 2 & 0.00030 & 1 & 1.46728 & 1.88700 & 17 & 0.282625 & 28 & 0.282622 & 8.9 & 1.0 & 1.02 & 658 & 10 & 99 \\
\hline zA15 & $\mathrm{m}$ & 0.0073 & 1 & 0.00031 & 0 & 1.46731 & 1.88684 & 20 & 0.282626 & 28 & 0.282622 & 8.6 & 1.0 & 1.03 & 644 & 10 & 98 \\
\hline zA16 & $\mathrm{m}$ & 0.0079 & 1 & 0.00029 & 0 & 1.46723 & 1.88682 & 15 & 0.282644 & 29 & 0.282640 & 9.1 & 1.0 & 1.00 & 637 & 12 & 95 \\
\hline zA17 & $\mathrm{m}$ & 0.0143 & 2 & 0.00050 & 1 & 1.46728 & 1.88681 & 17 & 0.282631 & 28 & 0.282625 & 8.4 & 1.0 & 1.03 & 631 & 9 & 95 \\
\hline zA18 & $\mathrm{m}$ & 0.0082 & 1 & 0.00032 & 0 & 1.46728 & 1.88683 & 17 & 0.282633 & 27 & 0.282630 & 8.3 & 0.9 & 1.03 & 619 & 11 & 98 \\
\hline zA19 & $\mathrm{m}$ & 0.0145 & 5 & 0.00052 & 2 & 1.46727 & 1.88671 & 16 & 0.282618 & 29 & 0.282612 & 7.9 & 1.0 & 1.06 & 627 & 18 & 93 \\
\hline $\mathrm{zA} 20$ & $\mathrm{~m}$ & 0.0151 & 13 & 0.00053 & 4 & 1.46729 & 1.88688 & 15 & 0.282640 & 29 & 0.282633 & 9.2 & 1.0 & 1.01 & 651 & 12 & 100 \\
\hline $\mathrm{zA} 21$ & $\mathrm{~m}$ & 0.0184 & 19 & 0.00065 & 7 & 1.46726 & 1.88683 & 15 & 0.282615 & 31 & 0.282607 & 8.3 & 1.1 & 1.06 & 652 & 11 & 101 \\
\hline zA22 & $\mathrm{m}$ & 0.0123 & 3 & 0.00043 & 1 & 1.46723 & 1.88669 & 15 & 0.282614 & 36 & 0.282608 & 8.0 & 1.3 & 1.06 & 637 & 9 & 98 \\
\hline zA23 & $\mathrm{m}$ & 0.0154 & 5 & 0.00055 & 2 & 1.46726 & 1.88673 & 15 & 0.282620 & 31 & 0.282613 & 8.4 & 1.1 & 1.05 & 650 & 11 & 99 \\
\hline
\end{tabular}




\section{Continued}

\begin{tabular}{lllllllllllllllllll}
\hline $\mathrm{zA} 24$ & $\mathrm{~m}$ & 0.0132 & 4 & 0.00047 & 2 & 1.46725 & 1.88675 & 16 & 0.282619 & 28 & 0.282613 & 8.5 & 1.0 & 1.04 & 653 & 11 & 100 \\
$\mathrm{zA} 25$ & $\mathrm{~m}$ & 0.0177 & 5 & 0.00067 & 2 & 1.46725 & 1.88666 & 15 & 0.282615 & 28 & 0.282607 & 8.0 & 1.0 & 1.06 & 638 & 11 & 94
\end{tabular}

(a) ${ }^{176} \mathrm{Yb} /{ }^{177} \mathrm{Hf}=\left({ }^{176} \mathrm{Yb} /{ }^{173} \mathrm{Yb}\right)_{\text {true }} \times\left({ }^{173} \mathrm{Yb} /{ }^{177} \mathrm{Hf}\right)_{\text {meas }} \times\left(\mathrm{M}_{173(\mathrm{Yb})} / \mathrm{M}_{177(\mathrm{Hf})}\right){ }^{\mathrm{b}(\mathrm{Hf})}, \mathrm{b}(\mathrm{Hf})=\ln \left({ }^{179} \mathrm{Hf} /{ }^{177} \mathrm{Hf}\right.$ true $/{ }^{179} \mathrm{Hf} /{ }^{177} \mathrm{Hf}$ measured $) / \ln (\mathrm{M} 179(\mathrm{Hf}) / \mathrm{M} 177(\mathrm{Hf}))$.

$\mathrm{M}=$ mass of respective isotope. The ${ }^{176} \mathrm{Lu} /{ }^{177} \mathrm{Hf}$ were calculated in a similar way by using the ${ }^{175} \mathrm{Lu} /{ }^{177} \mathrm{Hf}$ and $\mathrm{b}(\mathrm{Yb})$.

Quoted uncertainties (absolute) relate to the last quoted figure. The effect of the inter-element fractionation on the Lu/Hf was estimated to be about $6 \%$ or less based on analyses of the GJ-1 and Plesoviče zircons.

(b) Mean Hf signal in volt.

(c) Uncertainties are quadratic additions of the within-run precision and the daily reproducibility of the zircon GJ-1. Uncertainties for GJ-1 is 2SD (2 standard deviation).

(d) Initial ${ }^{176} \mathrm{Hf} /{ }^{177} \mathrm{Hf}$ and $\mathrm{Hf}$ calculated using the age (Ma). and the CHUR parameters:

${ }^{176} \mathrm{Lu} /{ }^{177} \mathrm{Hf}=0.0336$. and ${ }^{176} \mathrm{Hf} /{ }^{177} \mathrm{Hf}=0.282785$ (Bouvier et al. 2008).

(e) two stage model age in billion years using the measured ${ }^{176} \mathrm{Lu} /{ }^{177} \mathrm{Lu}$.thestimated age (Ma). a value of 0.01113 for the averageconinental crust (second stage). and a depleted mantle ${ }^{176} \mathrm{Lu} /{ }^{177} \mathrm{Hf}$ and ${ }^{176} \mathrm{Hf} /{ }^{177} \mathrm{Hf}$ of 0.03933 and 0.283294 .

(f) ${ }^{206} \mathrm{~Pb} /{ }^{238} \mathrm{U}$ age for zircon $<1.0 \mathrm{Ga}$. and ${ }^{206} \mathrm{~Pb} /{ }^{207} \mathrm{~Pb}$ age for zircon $>1.0 \mathrm{Ga}$.

(g) degree of concordance

\begin{tabular}{|c|c|c|c|c|c|c|c|c|c|c|c|c|c|c|}
\hline \multicolumn{15}{|c|}{ zircon standard GJ1 } \\
\hline GJ1-40-1 & 0.0066 & 0.5 & 0.00023 & 0.04 & 1.46725 & 1.88628 & 8 & 0.281995 & 29 & 0.281992 & -14.5 & 1.0 & 2.27 & 606 \\
\hline GJ1-40-2 & 0.0068 & 0.5 & 0.00024 & 0.03 & 1.46725 & 1.88619 & 8 & 0.282012 & 34 & 0.282010 & -13.9 & 1.2 & 2.24 & 606 \\
\hline GJ1-40-3 & 0.0071 & 0.6 & 0.00025 & 0.05 & 1.46731 & 1.88609 & 8 & 0.282025 & 33 & 0.282023 & -13.5 & 1.2 & 2.21 & 606 \\
\hline GJ1-40-4 & 0.0068 & 0.4 & 0.00025 & 0.05 & 1.46729 & 1.88633 & 7 & 0.282009 & 34 & 0.282006 & -14.0 & 1.2 & 2.24 & 606 \\
\hline GJ1-40-5 & 0.0069 & 0.2 & 0.00025 & 0.04 & 1.46726 & 1.88621 & 7 & 0.282005 & 33 & 0.282002 & -14.2 & 1.2 & 2.25 & 606 \\
\hline GJ1-40-6 & 0.0068 & 0.6 & 0.00024 & 0.06 & 1.46732 & 1.88640 & 8 & 0.282012 & 35 & 0.282009 & -13.9 & 1.2 & 2.24 & 606 \\
\hline GJ1-40-7 & 0.0074 & 1.0 & 0.00025 & 0.04 & 1.46735 & 1.88647 & 12 & 0.282009 & 29 & 0.282006 & -14.0 & 1.0 & 2.24 & 606 \\
\hline GJ1-40-9 & 0.0073 & 0.7 & 0.00025 & 0.05 & 1.46724 & 1.88642 & 12 & 0.282002 & 29 & 0.281999 & -14.3 & 1.0 & 2.25 & 606 \\
\hline GJ1-40-10 & 0.0069 & 0.4 & 0.00025 & 0.06 & 1.46717 & 1.88647 & 6 & 0.281990 & 39 & 0.281987 & -14.7 & 1.4 & 2.28 & 606 \\
\hline GJ1-40-11 & 0.0068 & 0.4 & 0.00025 & 0.08 & 1.46720 & 1.88643 & 6 & 0.281994 & 31 & 0.281992 & -14.6 & 1.1 & 2.27 & 606 \\
\hline GJ1-40-12 & 0.0069 & 0.3 & 0.00025 & 0.05 & 1.46729 & 1.88668 & 11 & 0.281981 & 30 & 0.281978 & -15.0 & 1.1 & 2.30 & 606 \\
\hline GJ1-40-13 & 0.0070 & 0.4 & 0.00024 & 0.02 & 1.46727 & 1.88632 & 14 & 0.281982 & 32 & 0.281980 & -15.0 & 1.1 & 2.29 & 606 \\
\hline GJ1-40-14 & 0.0070 & 0.3 & 0.00025 & 0.03 & 1.46730 & 1.88643 & 13 & 0.282002 & 31 & 0.281999 & -14.3 & 1.1 & 2.26 & 606 \\
\hline GJ1-40-15 & 0.0071 & 0.3 & 0.00025 & 0.03 & 1.46735 & 1.88640 & 14 & 0.281986 & 29 & 0.281983 & -14.9 & 1.0 & 2.29 & 606 \\
\hline GJ1-40-16 & 0.0068 & 0.3 & 0.00025 & 0.06 & 1.46726 & 1.88643 & 12 & 0.282001 & 29 & 0.281998 & -14.3 & 1.0 & 2.26 & 606 \\
\hline GJ1-40-17 & 0.0068 & 0.2 & 0.00025 & 0.05 & 1.46725 & 1.88623 & 6 & 0.282010 & 35 & 0.282008 & -14.0 & 1.3 & 2.24 & 606 \\
\hline GJ1-40-19 & 0.0070 & 0.5 & 0.00025 & 0.04 & 1.46730 & 1.88638 & 12 & 0.281992 & 31 & 0.281989 & -14.6 & 1.1 & 2.27 & 606 \\
\hline $\begin{array}{c}\text { mean } \\
(\mathrm{n}=19)\end{array}$ & 0.0070 & & 0.00025 & & 1.46728 & 1.88636 & & & & 0.281997 & -14.4 & & & \\
\hline 2 S.D. & 0.0004 & & 0.00001 & & 0.00009 & 0.00029 & & & & 0.000023 & 0.8 & & & \\
\hline
\end{tabular}


A. N. Saha-Fouotsa et al.

\section{Continued}

\begin{tabular}{|c|c|c|c|c|c|c|c|c|c|c|c|c|c|c|}
\hline \multicolumn{15}{|c|}{ zircon standard Plesovice } \\
\hline Pleso1 & 0.0039 & 0.2 & 0.00010 & 0.06 & 1.46729 & 1.88636 & 11 & 0.282441 & 28 & 0.282440 & -4.7 & 1.0 & 1.52 & 337 \\
\hline Pleso2 & 0.0041 & 0.3 & 0.00010 & 0.09 & 1.46729 & 1.88646 & 11 & 0.282453 & 29 & 0.282452 & -4.3 & 1.0 & 1.50 & 337 \\
\hline Pleso3 & 0.0039 & 0.1 & 0.00009 & 0.04 & 1.46731 & 1.88651 & 12 & 0.282434 & 29 & 0.282434 & -4.9 & 1.0 & 1.53 & 337 \\
\hline Pleso4 & 0.0041 & 1.0 & 0.00010 & 0.26 & 1.46737 & 1.88658 & 25 & 0.282494 & 26 & 0.282494 & -2.8 & 0.9 & 1.42 & 337 \\
\hline Pleso5 & 0.0039 & 1.2 & 0.00010 & 0.29 & 1.46727 & 1.88638 & 10 & 0.282449 & 33 & 0.282448 & -4.4 & 1.2 & 1.51 & 337 \\
\hline Pleso6 & 0.0038 & 1.0 & 0.00009 & 0.25 & 1.46733 & 1.88636 & 11 & 0.282471 & 31 & 0.282470 & -3.6 & 1.1 & 1.46 & 337 \\
\hline Pleso7 & 0.0055 & 0.6 & 0.00013 & 0.09 & 1.46735 & 1.88673 & 16 & 0.282479 & 29 & 0.282478 & -3.3 & 1.0 & 1.45 & 337 \\
\hline Pleso8 & 0.0040 & 0.6 & 0.00010 & 0.12 & 1.46735 & 1.88651 & 17 & 0.282490 & 29 & 0.282489 & -3.0 & 1.0 & 1.43 & 337 \\
\hline $\begin{array}{l}\text { mean } \\
(\mathrm{n}=8)\end{array}$ & 0.0041 & & 0.00010 & & 1.46732 & 1.88649 & & & & 0.282463 & -3.9 & & & \\
\hline 2 S.D. & 0.0011 & & 0.00002 & & 0.00007 & 0.00025 & & & & 0.000046 & 1.6 & & & \\
\hline \multicolumn{15}{|c|}{ zircon standard 91500} \\
\hline $91500-1$ & 0.0086 & 1 & 0.00031 & 0.2 & 1.46736 & 1.88651 & 5 & 0.282302 & 39 & 0.282296 & 6.6 & 1.4 & 1.47 & 1065 \\
\hline $91500-2$ & 0.0087 & 1 & 0.00031 & 0.3 & 1.46724 & 1.88630 & 7 & 0.282327 & 37 & 0.282321 & 7.5 & 1.3 & 1.43 & 1065 \\
\hline $91500-3$ & 0.0089 & 1 & 0.00032 & 0.2 & 1.46731 & 1.88665 & 14 & 0.282288 & 30 & 0.282282 & 6.1 & 1.1 & 1.50 & 1065 \\
\hline $91500-4$ & 0.0091 & 1 & 0.00032 & 0.3 & 1.46741 & 1.88664 & 12 & 0.282326 & 30 & 0.282319 & 7.4 & 1.1 & 1.43 & 1065 \\
\hline $91500-5$ & 0.0093 & 2 & 0.00033 & 0.5 & 1.46730 & 1.88643 & 12 & 0.282323 & 28 & 0.282316 & 7.3 & 1.0 & 1.43 & 1065 \\
\hline $91500-6$ & 0.0091 & 1 & 0.00030 & 0.1 & 1.46728 & 1.88644 & 9 & 0.282293 & 30 & 0.282287 & 6.3 & 1.1 & 1.49 & 1065 \\
\hline $91500-7$ & 0.0090 & 1 & 0.00031 & 0.3 & 1.46733 & 1.88660 & 9 & 0.282325 & 32 & 0.282319 & 7.4 & 1.1 & 1.43 & 1065 \\
\hline $\begin{array}{l}\text { mean } \\
(\mathrm{n}=7)\end{array}$ & 0.0089 & & 0.00031 & & 1.46732 & 1.88651 & & & & 0.282306 & 6.9 & & & \\
\hline 2 S.D. & 0.0005 & & 0.00002 & & 0.00011 & 0.00026 & & & & 0.000034 & 1.2 & & & \\
\hline \multicolumn{15}{|c|}{ zircon standard Temora } \\
\hline Temoral & 0.0214 & 5 & 0.00077 & 1.7 & 1.46729 & 1.88665 & 8 & 0.282671 & 32 & 0.282665 & 3.7 & 1.1 & 1.13 & 419 \\
\hline Temora2 & 0.0291 & 3 & 0.00104 & 1.1 & 1.46731 & 1.88659 & 8 & 0.282664 & 32 & 0.282656 & 3.4 & 1.1 & 1.14 & 419 \\
\hline Temora3 & 0.0130 & 4 & 0.00047 & 1.6 & 1.46727 & 1.88666 & 9 & 0.282677 & 31 & 0.282674 & 4.0 & 1.1 & 1.11 & 419 \\
\hline Temora4 & 0.0232 & 0 & 0.00080 & 0.2 & 1.46735 & 1.88670 & 8 & 0.282693 & 33 & 0.282687 & 4.5 & 1.2 & 1.08 & 419 \\
\hline Temora5 & 0.0278 & 18 & 0.00096 & 6.4 & 1.46735 & 1.88619 & 7 & 0.282692 & 37 & 0.282684 & 4.4 & 1.3 & 1.09 & 419 \\
\hline Temora6 & 0.0324 & 11 & 0.00115 & 3.4 & 1.46737 & 1.88654 & 7 & 0.282692 & 36 & 0.282683 & 4.3 & 1.3 & 1.09 & 419 \\
\hline Temora7 & 0.0237 & 4 & 0.00077 & 1.7 & 1.46733 & 1.88654 & 6 & 0.282694 & 41 & 0.282688 & 4.5 & 1.4 & 1.08 & 419 \\
\hline $\begin{array}{c}\text { mean } \\
(\mathrm{n}=7)\end{array}$ & 0.0244 & & 0.00085 & & 1.46732 & 1.88655 & & & & 0.282677 & 4.1 & & & \\
\hline 2 S.D. & 0.0126 & & 0.00044 & & 0.00007 & 0.00034 & & & & 0.000025 & 0.9 & & & \\
\hline
\end{tabular}

results of standard measurements 23-01-2015

\begin{tabular}{llllllllllllllllllll}
\hline \multicolumn{2}{c}{ zircon standard GJ1 } \\
\hline GJ-50-1 & 0.0068 & 0.4 & 0.00025 & 0.06 & 1.46727 & 1.88670 & 16 & 0.282013 & 35 & 0.282011 & -13.9 & 1.2 & 2.23 & 606 & 1 & \\
GJ-50-2 & 0.0068 & 0.3 & 0.00025 & 0.05 & 1.46729 & 1.88696 & 16 & 0.282000 & 35 & 0.281997 & -14.4 & 1.2 & 2.26 & 606 & 1 & \\
GJ-50-3 & 0.0069 & 0.3 & 0.00025 & 0.05 & 1.46723 & 1.88674 & 17 & 0.281998 & 37 & 0.281995 & -14.4 & 1.3 & 2.26 & 606 & 1 & \\
\hline
\end{tabular}




\section{Continued}

\begin{tabular}{|c|c|c|c|c|c|c|c|c|c|c|c|c|c|c|c|}
\hline GJ-50-4 & 0.0069 & 0.4 & 0.00025 & 0.06 & 1.46731 & 1.88677 & 16 & 0.281981 & 33 & 0.281979 & -15.0 & 1.2 & 2.29 & 606 & 1 \\
\hline GJ-50-5 & 0.0068 & 0.3 & 0.00025 & 0.05 & 1.46729 & 1.88677 & 16 & 0.282010 & 34 & 0.282007 & -14.0 & 1.2 & 2.24 & 606 & 1 \\
\hline GJ-50-7 & 0.0069 & 0.3 & 0.00025 & 0.05 & 1.46735 & 1.88675 & 12 & 0.282022 & 36 & 0.282019 & -13.6 & 1.3 & 2.22 & 606 & 1 \\
\hline GJ-50-8 & 0.0069 & 0.2 & 0.00025 & 0.05 & 1.46735 & 1.88676 & 12 & 0.282029 & 35 & 0.282027 & -13.3 & 1.2 & 2.20 & 606 & 1 \\
\hline GJ-50-9 & 0.0070 & 0.3 & 0.00025 & 0.04 & 1.46732 & 1.88683 & 13 & 0.282013 & 34 & 0.282010 & -13.9 & 1.2 & 2.23 & 606 & 1 \\
\hline GJ-50-12 & 0.0070 & 0.4 & 0.00025 & 0.04 & 1.46725 & 1.88689 & 9 & 0.281992 & 36 & 0.281990 & -14.6 & 1.3 & 2.27 & 606 & 1 \\
\hline GJ-50-13 & 0.0069 & 0.3 & 0.00025 & 0.06 & 1.46722 & 1.88660 & 9 & 0.281988 & 36 & 0.281985 & -14.8 & 1.3 & 2.28 & 606 & 1 \\
\hline GJ-50-14 & 0.0070 & 0.2 & 0.00025 & 0.04 & 1.46717 & 1.88680 & 9 & 0.281973 & 38 & 0.281970 & -15.3 & 1.4 & 2.31 & 606 & 1 \\
\hline GJ-50-16 & 0.0071 & 0.5 & 0.00025 & 0.06 & 1.46728 & 1.88667 & 9 & 0.281999 & 35 & 0.281996 & -14.4 & 1.2 & 2.26 & 606 & 1 \\
\hline GJ-50-17 & 0.0070 & 0.4 & 0.00025 & 0.06 & 1.46722 & 1.88668 & 9 & 0.282004 & 40 & 0.282002 & -14.2 & 1.4 & 2.25 & 606 & 1 \\
\hline GJ-50-18 & 0.0069 & 0.3 & 0.00024 & 0.06 & 1.46725 & 1.88679 & 9 & 0.281987 & 35 & 0.281984 & -14.8 & 1.2 & 2.28 & 606 & 1 \\
\hline GJ-50-19 & 0.0070 & 0.3 & 0.00025 & 0.04 & 1.46721 & 1.88672 & 9 & 0.282017 & 37 & 0.282014 & -13.8 & 1.3 & 2.23 & 606 & 1 \\
\hline GJ-50-20 & 0.0070 & 0.3 & 0.00025 & 0.05 & 1.46727 & 1.88679 & 9 & 0.281973 & 39 & 0.281970 & -15.3 & 1.4 & 2.31 & 606 & 1 \\
\hline GJ-50-21 & 0.0070 & 0.5 & 0.00025 & 0.05 & 1.46724 & 1.88670 & 9 & 0.281984 & 35 & 0.281981 & -14.9 & 1.2 & 2.29 & 606 & 1 \\
\hline GJ-50-22 & 0.0070 & 0.4 & 0.00025 & 0.04 & 1.46727 & 1.88659 & 8 & 0.282006 & 38 & 0.282003 & -14.1 & 1.3 & 2.25 & 606 & 1 \\
\hline GJ-50-23 & 0.0070 & 0.3 & 0.00025 & 0.06 & 1.46721 & 1.88676 & 8 & 0.282013 & 35 & 0.282010 & -13.9 & 1.2 & 2.23 & 606 & 1 \\
\hline GJ-50-24 & 0.0071 & 0.4 & 0.00025 & 0.06 & 1.46726 & 1.88662 & 8 & 0.282007 & 36 & 0.282004 & -14.1 & 1.3 & 2.25 & 606 & 1 \\
\hline GJ-50-26 & 0.0071 & 0.4 & 0.00025 & 0.07 & 1.46725 & 1.88657 & 8 & 0.281997 & 39 & 0.281994 & -14.5 & 1.4 & 2.26 & 606 & 1 \\
\hline GJ-50-27 & 0.0072 & 0.3 & 0.00026 & 0.04 & 1.46730 & 1.88664 & 9 & 0.282009 & 37 & 0.282006 & -14.1 & 1.3 & 2.24 & 606 & 1 \\
\hline $\begin{array}{c}\text { mean } \\
(\mathrm{n}=27)\end{array}$ & 0.0070 & & 0.00025 & & 1.46726 & 1.88673 & & 0.282000 & & 0.281997 & -14.4 & & & & \\
\hline 2 S.D. & 0.0002 & & 0.00001 & & 0.00010 & 0.00018 & & 0.000030 & & 0.000030 & 1.1 & & & & \\
\hline \multicolumn{16}{|c|}{ zircon standard Plesovice } \\
\hline Pleso1 & 0.0042 & 0.3 & 0.00010 & 0.0 & 1.46724 & 1.88675 & 12 & 0.282457 & 37 & 0.282456 & -5.2 & 1.3 & 1.55 & 337 & 1 \\
\hline Pleso2 & 0.0042 & 0.5 & 0.00010 & 0.0 & 1.46727 & 1.88676 & 11 & 0.282484 & 36 & 0.282483 & -4.2 & 1.3 & 1.50 & 337 & 1 \\
\hline Pleso3 & 0.0043 & 0.5 & 0.00010 & 0.1 & 1.46718 & 1.88668 & 11 & 0.282462 & 35 & 0.282461 & -5.0 & 1.2 & 1.54 & 337 & 1 \\
\hline Pleso4 & 0.0046 & 0.1 & 0.00011 & 0.1 & 1.46727 & 1.88675 & 24 & 0.282472 & 32 & 0.282471 & -4.7 & 1.1 & 1.52 & 337 & 1 \\
\hline Pleso5 & 0.0033 & 0.2 & 0.00008 & 0.1 & 1.46719 & 1.88680 & 14 & 0.282468 & 34 & 0.282467 & -4.8 & 1.2 & 1.53 & 337 & 1 \\
\hline Pleso6 & 0.0041 & 0.1 & 0.00009 & 0.0 & 1.46722 & 1.88690 & 15 & 0.282474 & 34 & 0.282473 & -4.6 & 1.2 & 1.51 & 337 & 1 \\
\hline Pleso7 & 0.0056 & 0.7 & 0.00013 & 0.2 & 1.46720 & 1.88679 & 15 & 0.282466 & 34 & 0.282465 & -4.9 & 1.2 & 1.53 & 337 & 1 \\
\hline Pleso8 & 0.0061 & 0.7 & 0.00015 & 0.2 & 1.46723 & 1.88679 & 13 & 0.282496 & 34 & 0.282495 & -3.8 & 1.2 & 1.47 & 337 & 1 \\
\hline Pleso9 & 0.0062 & 0.9 & 0.00015 & 0.2 & 1.46727 & 1.88668 & 13 & 0.282485 & 37 & 0.282484 & -4.2 & 1.3 & 1.49 & 337 & 1 \\
\hline Pleso10 & 0.0060 & 0.7 & 0.00015 & 0.2 & 1.46723 & 1.88685 & 13 & 0.282472 & 34 & 0.282472 & -4.7 & 1.2 & 1.52 & 337 & 1 \\
\hline Pleso11 & 0.0044 & 0.2 & 0.00010 & 0.0 & 1.46729 & 1.88676 & 12 & 0.282512 & 37 & 0.282511 & -3.2 & 1.3 & 1.44 & 337 & 1 \\
\hline Pleso12 & 0.0043 & 0.1 & 0.00010 & 0.0 & 1.46724 & 1.88676 & 12 & 0.282508 & 34 & 0.282507 & -3.4 & 1.2 & 1.45 & 337 & 1 \\
\hline
\end{tabular}




\section{Continued}

\begin{tabular}{|c|c|c|c|c|c|c|c|c|c|c|c|c|c|c|c|}
\hline Pleso13 & 0.0043 & 0.2 & 0.00010 & 0.0 & 1.46730 & 1.88684 & 13 & 0.282487 & 36 & 0.282486 & -4.1 & 1.3 & 1.49 & 337 & 1 \\
\hline Pleso14 & 0.0043 & 0.6 & 0.00010 & 0.1 & 1.46728 & 1.88692 & 12 & 0.282508 & 35 & 0.282507 & -3.4 & 1.2 & 1.45 & 337 & 1 \\
\hline Pleso15 & 0.0041 & 0.7 & 0.00010 & 0.1 & 1.46732 & 1.88691 & 12 & 0.282518 & 36 & 0.282517 & -3.0 & 1.3 & 1.43 & 337 & 1 \\
\hline Pleso16 & 0.0039 & 0.8 & 0.00009 & 0.2 & 1.46725 & 1.88672 & 12 & 0.282492 & 37 & 0.282492 & -3.9 & 1.3 & 1.48 & 337 & 1 \\
\hline $\begin{array}{c}\text { mean } \\
(n=16)\end{array}$ & 0.0046 & & 0.00011 & & 1.46725 & 1.88679 & & 0.282485 & & 0.282484 & -4.2 & & & & \\
\hline 2 S.D. & 0.0017 & & 0.00004 & & 0.00008 & 0.00015 & & 0.000038 & & 0.000038 & 1.3 & & & & \\
\hline \multicolumn{16}{|c|}{ zircon standard 91500} \\
\hline $91500-1$ & 0.0097 & 2 & 0.00032 & 0.3 & 1.46729 & 1.88677 & 13 & 0.282274 & 35 & 0.282268 & 5.6 & 1.2 & 1.53 & 1065 & 1 \\
\hline $91500-2$ & 0.0100 & 2 & 0.00033 & 0.5 & 1.46731 & 1.88683 & 12 & 0.282293 & 36 & 0.282287 & 6.3 & 1.3 & 1.49 & 1065 & 1 \\
\hline $91500-4$ & 0.0095 & 1 & 0.00032 & 0.3 & 1.46720 & 1.88686 & 7 & 0.282267 & 38 & 0.282261 & 5.3 & 1.4 & 1.54 & 1065 & 1 \\
\hline $91500-5$ & 0.0095 & 1 & 0.00032 & 0.1 & 1.46725 & 1.88654 & 8 & 0.282293 & 37 & 0.282287 & 6.3 & 1.3 & 1.49 & 1065 & 1 \\
\hline $91500-6$ & 0.0096 & 1 & 0.00031 & 0.2 & 1.46717 & 1.88661 & 8 & 0.282269 & 38 & 0.282262 & 5.4 & 1.4 & 1.54 & 1065 & 1 \\
\hline $91500-7$ & 0.0094 & 2 & 0.00031 & 0.3 & 1.46720 & 1.88655 & 7 & 0.282288 & 39 & 0.282282 & 6.1 & 1.4 & 1.50 & 1065 & 1 \\
\hline $91500-8$ & 0.0093 & 1 & 0.00030 & 0.1 & 1.46717 & 1.88642 & 8 & 0.282280 & 38 & 0.282274 & 5.8 & 1.4 & 1.52 & 1065 & 1 \\
\hline $\begin{array}{c}\text { mean } \\
(n=8)\end{array}$ & 0.0096 & & 0.00032 & & 1.46724 & 1.88668 & & 0.282280 & & 0.282274 & 5.8 & & & & \\
\hline 2 S.D. & 0.0005 & & 0.00002 & & 0.00012 & 0.00035 & & 0.000021 & & 0.000021 & 0.7 & & & & \\
\hline \multicolumn{16}{|c|}{ zircon standard Temora } \\
\hline Temora1 & 0.0221 & 2 & 0.00078 & 0.9 & 1.46725 & 1.88655 & 8 & 0.282643 & 39 & 0.282637 & 4.1 & 1.4 & 1.10 & 419 & 1 \\
\hline Temora3 & 0.0292 & 3 & 0.00095 & 0.5 & 1.46728 & 1.88649 & 9 & 0.282648 & 40 & 0.282641 & 4.2 & 1.4 & 1.10 & 419 & 1 \\
\hline Temora4 & 0.0192 & 3 & 0.00068 & 1.1 & 1.46715 & 1.88651 & 5 & 0.282672 & 47 & 0.282667 & 5.2 & 1.6 & 1.04 & 419 & 1 \\
\hline Temora5 & 0.0232 & 10 & 0.00081 & 3.3 & 1.46712 & 1.88680 & 5 & 0.282691 & 48 & 0.282684 & 5.8 & 1.7 & 1.01 & 419 & 1 \\
\hline Temora6 & 0.0271 & 8 & 0.00094 & 2.3 & 1.46716 & 1.88669 & 5 & 0.282658 & 43 & 0.282650 & 4.6 & 1.5 & 1.08 & 419 & 1 \\
\hline Temora7 & 0.0334 & 5 & 0.00108 & 1.5 & 1.46728 & 1.88687 & 10 & 0.282650 & 37 & 0.282642 & 4.3 & 1.3 & 1.09 & 419 & 1 \\
\hline Temora8 & 0.0175 & 2 & 0.00058 & 0.9 & 1.46727 & 1.88666 & 11 & 0.282642 & 35 & 0.282637 & 4.1 & 1.2 & 1.10 & 419 & 1 \\
\hline $\begin{array}{c}\text { mean } \\
(n=8)\end{array}$ & 0.0239 & & 0.00081 & & 1.46722 & 1.88667 & & 0.282656 & & 0.282650 & 4.6 & & & & \\
\hline 2 S.D. & 0.0111 & & 0.00033 & & 0.00013 & 0.00027 & & 0.000034 & & 0.000034 & 1.2 & & & & \\
\hline
\end{tabular}

\section{LA-MC-ICPMS Lu-Hf Result of the Tonalite}

The studied tonalite sample is representative of a kilometre-scale pluton elongated in the HT foliation at the regional scale. Zircon grains from this sample are mainly subhedral, prismatic with slightly rounded terminations. The grain size varies from 200 to $500 \mu \mathrm{m}$ in length, and CL images show oscillatory zoning suggesting a magmatic origin (Figure 4). Taking into account the large size of the grains and the texture of the zircon analysed, several points of analysis were made on mineral cores and edges to have a better analysis and interpretation of the ages obtained. 


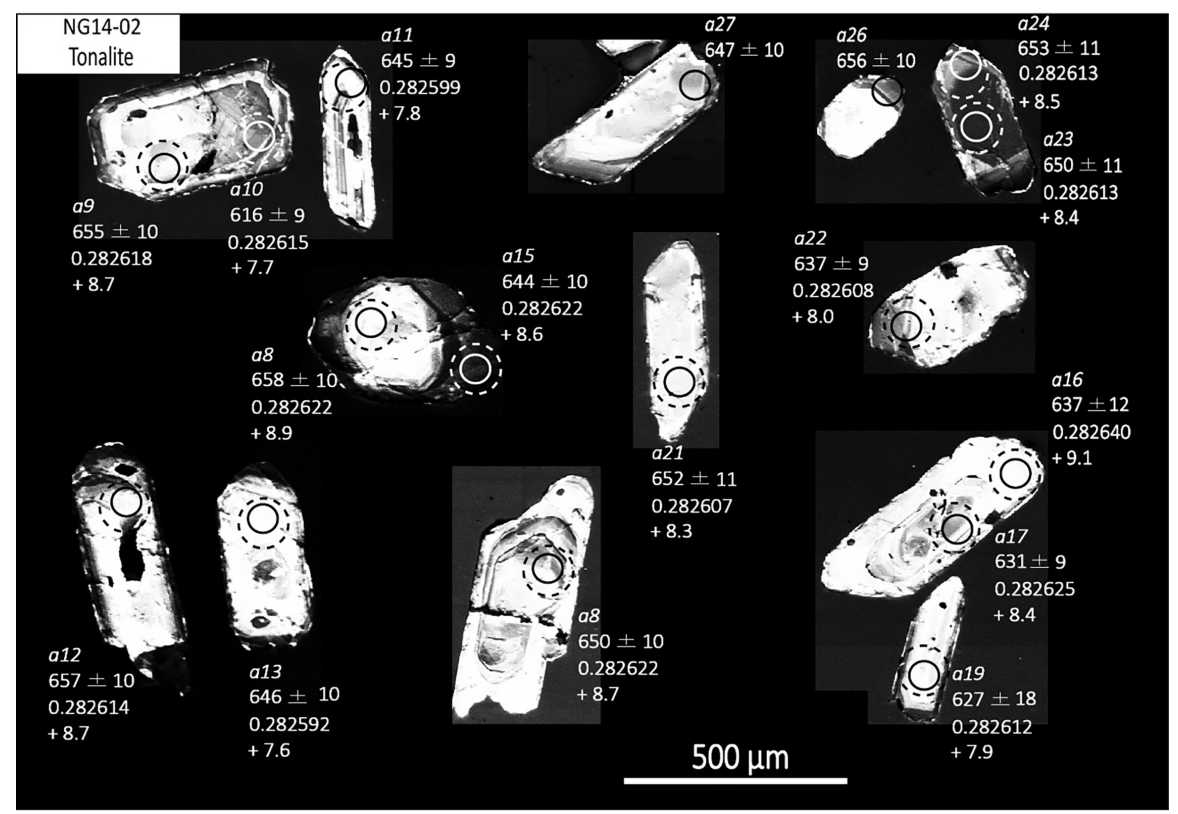

Figure 4. Cathodoluminescence (CL) images of analysed tonalite representative zircons of Mbé - Sassa-Mbersi region. The solid circle shows the location of U-Pb isotopic analysis spot and the dashed circle indicates the location of Lu-Hf isotopic analysis spot. The indications reported to de zircon image represent from top to bottom; the analysis numbers correspond to those in Table 1 , the $\mathrm{U}-\mathrm{Pb}$ ages, the ${ }^{176} \mathrm{Hf} /{ }^{177} \mathrm{Hf}(\mathrm{t})$ values and the $\varepsilon \mathrm{Hf}$ values.

Eighteen analyses were thus made on eleven grains of zircon yielding ${ }^{176} \mathrm{Lu} /{ }^{177} \mathrm{Hf}$ ratios vary from 0.00027 to 0.00105 and the homogeneous ${ }^{176} \mathrm{Hf} /{ }^{177} \mathrm{Hf}$ ratios from 0.282592 to 0.282640 . The $\varepsilon H f(t)$ values and $T_{D M}$ ages varying from +7.6 to +8.9 and from 1.0 to $1.09 \mathrm{Ga}$, respectively (Table 1; Figure 4).

\section{Discussion}

The Tonian extensional events were recorded in the São Francisco Congo Craton and surroundings (Silva et al., 1995; Correa-Gomes \& Oliveira, 2000; Tupinambá et al., 2007). These events all mark by the mafic dyke were subdivided into two major phase of continental rifting. The early episode occurred between 1100 - $1000 \mathrm{Ma}$ (Tupinambá et al., 2007) and while the second episode was expressed between 930 - $900 \mathrm{Ma}$ (Silva et al., 1995; Correa-Gomes \& Oliveira, 2000; Tupinambá et al., 2007).

The Lu-Hf isotopic data of ca. 1.0 Ga from the Nguesseck tonalite in the Mbé-Sassa-Mbersi region belong to this early episode of continental rifting in the São Francisco Congo Craton. Age obtained in this work is comparable to concordant inherited age of $1.05 \mathrm{Ga}$, got by Djerossem et al. (2020) to the magmatic zircon core of biotite granite in the Ouaddaï (Chad) which is part of Central African Orogenic Belt and continuity of the Central Cameroon Domain (Djerossem et al., 2020, 2021).

Although some authors have interpreted the Tonian episode as failed attempts of dislocation of the Atlantica Paleoproterozoic continent (Renné et al., 1990; 
Schobbenhaus, 1996; Uhlein et al., 1998; Alvarenga et al., 2000; Tack et al., 2001), the $\mathrm{T}_{\mathrm{DM}}$ ages obtained by Da Silva Filho et al. (2000) in the northern edge of the Sergipano belt were interpreted as an accretion of some juvenile material to the continental lithosphere and suggesting either formation of oceanic crust or underplating of mafic magmas during active rifting. The local occurrence of C-type eclogites near the Pernambuco shear zone (PSZ) in the central domain of the Borborema province (Beurlen et al., 1992) and obduction of ultramafic rocks with pillow basalts in the Araguaia belt (Alvarenga et al., 2000) are in favour with existence of an oceanic crust form around the early and middle Neproterozoic.

Geochemical data (magnesian medium-K series, fractionated REE patterns with low heavy REE concentrations, volcanic arc granite ...) of the Nguesseck tonalite $(651 \pm 3 \mathrm{Ma})$ obtained by Saha-Fouotsa et al. (2019) in the Mbé Sassa-Mbersi region suggest a possible origin by partial melting of mafic material involving fractionation of amphibole and garnet. The Lu-Hf data of this work suggest that, this mafic material would have formed around $1 \mathrm{Ga}$ taking into account the $\mathrm{Hf}_{\mathrm{DM}}$ ages obtained on all of the zircon grains analysed in the studied sample (Table 1; Figure 4 and Figure 5).

Thus, taking into account the positive values of $\varepsilon \mathrm{Hf}(\mathrm{t})(+7.6$ to +8.9$)$ from tonalite analysed zircon grains, we can suggest that juvenile tonian magmatism episode would have happened at the northern edge of the Central Domain of the Central African Orogenic belt. Which can be corroborate by concordant U/Pb inherited age (1.05 Ga) obtained by Djerossem et al. (2020) in the Ouaddaï (Chad). Geochemical data of the Nguesseck tonalite got by Saha-Fouotsa et al. (2019) with Hf positive value (Figure 5), could suggest that $\mathrm{Hf}_{\mathrm{TDM}}$ ages ca. $1.0 \mathrm{Ga}$ obtained in this work, would therefore correspond to the age of extraction of the mafic magma at the origin of the protholite of this tonalite. These $\mathrm{Hf}_{\mathrm{TDM}}$ ages could characterize a Neoproterozoic rifting stage with oceanic crust formation. The latter would therefore have subducted during the convergent phase of the Pan-African/Brasiliano orogeny, partially melted and gave Nguesseck tonalite magma which crystallized at around $651 \mathrm{Ma}$ (Saha-Fouotsa et al., 2019).

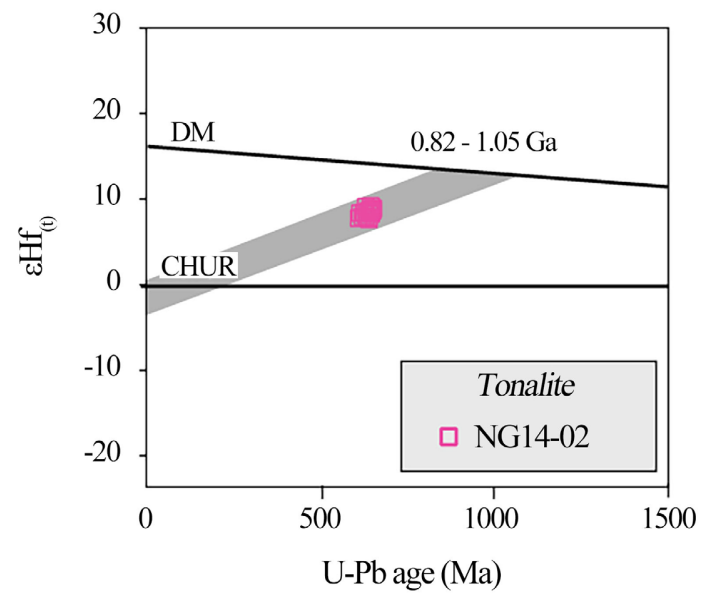

Figure 5. $\mathrm{U}-\mathrm{Pb}$ ages versus $\varepsilon \mathrm{Hf}(\mathrm{t})$ values diagram of analysed tonalite of Mbé-Sassa-Mbersi region. 
The time between $1.0 \mathrm{Ga}$ and $651 \mathrm{Ma}$ fits well into the estimated period for the formation of the old long-lived orogenic belts $(1000-600 \mathrm{Ma})$ to which the Central African Orogenic belt belongs (Kröner, 1975; Bayer \& Lesquer, 1978; Drake, 1980; Chikhaoui et al., 1980; Lesquer et al., 1981; Regan \& Marsh, 1982; Nzenti et al., 1984; Poidevin, 1985; Caby, 1987; Barbey et al., 1990; Fernandez et al., 1992; Penaye et al., 1993). Similar age (1011 $\pm 86 \mathrm{Ma}, 1051 \pm 22 \mathrm{Ma}, 1048 \pm$ $11 \mathrm{Ma}$ and $1056 \pm 21$ ) is obtained on zircons from alkaline syenitic gneisses by $\mathrm{Pb} / \mathrm{Pb}$ and $\mathrm{U} / \mathrm{Pb}$ method on zircon and interpreted as evidence of the beginning of the rifting process in the Araguaia Group in Brazil (Souza \& Moura 1995; Viana \& Battilani 2014).

\section{Conclusion}

Combined $\mathrm{Lu}-\mathrm{Hf}$ isotopic result obtained in this work $(\varepsilon \mathrm{Hf}(\mathrm{t})(+7.6$ to +8.9$)$ and $\mathrm{Hf}_{\mathrm{TDM}}$ ages ca. $1.0 \mathrm{Ga}$ ) and previous petrographical, geochemical and $\mathrm{U}-\mathrm{Pb}$ geochronological data suggest that the Nguessek tonalite would be the result of a partial melting of a $1.0 \mathrm{Ga}$ juvenile rock formed in a context of distancing at the beginning of the Neoproterozoic. This new data obtained in the Mbé-Sassa-Mbersi region, part of the Central Cameroon Domain which belongs to the $\mathrm{CAOB}$, allows to have other points of similarity between this orogenic bel and the Borborema Province in the NE of Brazil.

\section{Acknowledgements}

This work is integrated in the URCO research project financially supported by the CNRS-NEEDS program and the AUF program (Project $\mathrm{N}^{\circ}$ 51110SU201). We acknowledge fruitful discussions with Djerossem Nenadji Félix and Tsozué Désiré. Jean-François Ména is thanked for the help provided during the preparation of zircon grain for analysis at Geoscience Environment Toulouse laboratory (GET). The authors also thank the reviewers who made it possible to improve the quality of this paper.

\section{Conflicts of Interest}

The authors declare no conflicts of interest regarding the publication of this paper.

\section{References}

Alvarenga, C. J. S., Moura C. A. V., Gorayed P. S. S., \& Abreu F. A. M. (2000). Paraguay and Araguaia Belts. In U. G. Cordani et al. (Eds.), Tectonic Evolution of South America (pp. 183-193). International Union of Geological Sciences.

Barbey, P., Macaudière, J., \& Nzenti, J. P. (1990). High-Pressure Dehydration Melting of Metapelites: Evidence from the Migmatites of Yaoundé (Cameroun). Journal of Petrology, 31, 401-427. https://doi.org/10.1093/petrology/31.2.401

Bayer, R., \& Lesquer, A. (1978). Les anomalies gravimétiques de la bordure orientale du craton Ouest-africain: géométrie d'une suture panafricaine. Bulletin de la Société Géologique de France, 6, 868-876. https://doi.org/10.2113/gssgfbull.S7-XX.6.863 
Beurlen, H., Silva Filho, A. F., Guimarães, I. P., \& Brito, S. B. (1992). Proterozoic C-Type Eclogites Hosting Unusual $\mathrm{Ti}-\mathrm{Fe} \pm \mathrm{Cr} \pm \mathrm{Cu}$ Mineralization in Northeastern Brazil. Precambrian Research, 58, 195-214. https://doi.org/10.1016/0301-9268(92)90119-9

Bossière, G., Bonkoungou, I., Peucat, J. J., \& Pupin, J. P. (1996). Origin and Age of Paleoproterozoic Conglomerates and Sandstones of the Tarkwaian Group in Burkina Faso, West Africa. Precambrian Research, 80, 153-172. https://doi.org/10.1016/S0301-9268(96)00014-9

Bouvier, A., Vervoort, J. D., \& Patchett, P. J. (2008). The Lu-Hf and Sm-Nd Isotopic Composition of CHUR: Constraints from Unequilibrated Chondrites and Implications for the Bulk Composition of Terrestrial Planets. Earth and Planetary Science Letters, 273, 48-57. https://doi.org/10.1016/j.epsl.2008.06.010

Brito Neves, B. B., Campos Neto, M. C., Van Schmus, W. R., \& Santos, E. J. (2001). O Sistema Pajeú-Paraíba e o Macic, o São José de Campestre no leste da Borborema. $R e$ vista Brasileira Geociências, 31, 173-184. https://doi.org/10.25249/0375-7536.2001312173184

Caby, R. (1987). The Pan-African Belt of West Africa from the Sahara Desert to the Gulf of Benin. In J.P. Schaer, \& J. Rodgers (Eds.), Anatomy of Mountain Ranges (pp. 129-170). Princeton University Press. https://doi.org/10.1515/9781400858644.129

Chikhaoui, M., Dupuy, C., \& Dostal, J. (1980). Geochemistry and Petrogenesis of Late Proterozoic Volcanic Rocks from North-Western Africa. Contribution to Mineralogy and Petrology, 73, 375-388. https://doi.org/10.1007/BF00376630

Correa-Gomes, L. C., \& Oliveira, E. P. (2000). Radiating 1.0 Ga Mafic Dyke Swarms of Eastern Brazil an Western Africa: Evidence of Post-Assembly Extension in the Rodinia Supercontinent? Gondwana Research, 3, 325-332. https://doi.org/10.1016/S1342-937X(05)70291-4

Da Silva, Filho, A. F., Van Schmus, W. R., \& Guimarães, I. P. (2000). High-K Calc-Alkaline Granitoids of ca. $1 \mathrm{Ga}$ TDM along the Limit PE-AL Massif/Sergipano Fold Belt, NE Brazil, and Their Geotectonic Significance. Revista Brasileira de Geociências, 30, 182-185. https://doi.org/10.25249/0375-7536.2000301182185

Djerossem, F., Berger, J., Vanderhaeghe, O., Isseini, M., Ganne, J., \& Zeh, A. (2020). Neoproterozoic Magmatic Evolution of the Southern Ouaddaï Massif (Chad). EDP Sciences. https://www.bsgf.fr https://doi.org/10.1051/bsgf/2020032

Djerossem, F., Zeh, A., Isseini, M., Vanderhaeghe, O., Berger, J., \&. Ganne, J. (2021). $\mathrm{U}-\mathrm{Pb}$-Hf Isotopic Systematics of Zircon from Granite and Metasediments of Southern Ouaddaï (Chad), Implication for Crustal Evolution and Provenance in the Central African Orogenic Belt. Precambrian Research, 361, Article ID: 106233. https://doi.org/10.1016/j.precamres.2021.106233

Drake, A. A. (1980). Tectonic Studies in the Brazilian Shield. Professional Paper, 1119 A. TU.S. Government Publishing Office. https://doi.org/10.3133/pp1119AB

Fernandez, L. A. D., Tommasi, A., \& Porcher, C. C. (1992). Deformation Patterns in the Southern Brazilian Branch of the Dom Feliciano Belt: A Reappraisal. Journal of South American Earth Sciences, 5, 77-96. https://doi.org/10.1016/0895-9811(92)90061-3

Ganwa, A. A., Frisch, W., Siebel, W., Ekodeck, G. E., Shang, C. K., \& Ngako, V. (2008). Archean Inheritances in the Pyroxene-Amphibole Bearing Gneiss of the Méiganga Area (Central North Cameroon): Geochemical and 207Pb/206 Pb Age Imprints. Compte Rendu Geosciences, 340, 211-222. https://doi.org/10.1016/j.crte.2007.12.009

Ganwa, A. A., Siebel, W., Shang, C. K., Seguem, N., \& Ekodeck, G. E. (2011). New Constraints from $\mathrm{Pb}$-Evaporation Zircon Ages of the Méiganga Amphibole-Biotite Gneiss, 
Central Cameroon, on Proterozoic Crustal Evolution. International Journal of Geosciences, 2, 138-147. https://doi.org/10.4236/ijg.2011.22014

Gerdes, A., \& Zeh, A. (2006). Combined U-Pb and Hf Isotope LA-(MC) ICP-MS Analyses of Detrital Zircons: Comparison with SHRIMP and New Constraints for the Provenance and Age of an Armorican Metasediment in Central Germany. Earth and Planetary Science Letters, 249, 47-61. https://doi.org/10.1016/j.epsl.2006.06.039

Gerdes, A., \& Zeh, A. (2009). Zircon Formation versus Zircon Alteration-New Insights from Combined U-Pb and Lu-Hf in-Situ LA-ICP-MS Analyses, and Consequences for the Interpretation of Archaean Zircon from the Central Zone of the Limpopo Belt. Chemical Geology, 261, 230-243. https://doi.org/10.1016/j.chemgeo.2008.03.005

Gray, D. R., Foster, D. A., Meert, J. G., Goscombe, B. D., Armstrong, R. A., Trow, R. A., \& Passhier, C. W. (2008). A Damara Orogen Perspective on the Assembly of Southwestern Gondwana. Geological Society, 294, 257-278. https://doi.org/10.1144/SP294.14

Guimarães, I. P., Van Schmus, W. R., Brito Neves, B. B., Bretas Bittar, S. M., Silva Filho, A. F., \& Armstrong, R. (2012). U-Pb Zircon Ages of Orthogneisses and Supracrustal Rocks of the Cariris Velhos Belt: Onset of Neoproterozoic Rifting in the Borborema Province, NE Brazil. Precambrian Research, 192-195, 52-77.

https://doi.org/10.1016/j.precamres.2011.10.008

Kozuch, M. (2003). Isotopic and Trace Element Geochemistry of Early Neoproterozoic Gneissic and Metavolcanic rocks in the Cariris Velhos orogen of the Borborema Province, Brazil and their Bearing Tectonic Setting (199 p). PhD Thesis, Kansas University.

Kröner, A. (1975). Late Precambrian Formations in the Western Richtersveld, Northern Cape Province. Transactions of the Royal Society of South Africa, 41, 375-433. https://doi.org/10.1080/00359197509519451

Küster, D., \& Liégeois, J. P. (2001). Sr, Nd Isotopes and Geochemistry of the Bayuda Desert High-Grade Metamorphic Basement (Sudan): An Early Pan-African Oceanic Convergent Margin, Not the Edge of the East Saharan Ghost Craton? Precambrian Research, 109, 1-23. https://doi.org/10.1016/S0301-9268(00)00147-9

Kwékam, M., Liégeois, J.-P., Njonfang, E., Affaton, P., Hartman, G., \& Tchoua, F. (2010). Nature, Origin and Significance of the Fomopéa Pan-African High-K Calc-Alkaline Plutonic Complex in the Central African Fold Belt (Cameroun). Journal of African Earth Sciences, 54, 79-95. https://doi.org/10.1016/j.jafrearsci.2009.07.012

Ledru, P., Johan, V., Milési, J. P., \& Tegyey, M. (1994). Markers of the Last Stages of the Paleoproterozoic Collision: Evidence for a 2 Ga Continent Involving Circum-South Atlantic provinces. Precambrian Research, 69, 169-191. https://doi.org/10.1016/0301-9268(94)90085-X

Lesquer, A., De Almeida, F. F. M., Davino, A., Lachaud, J. C., \& Maillard, P. (1981). Signification structurale des anomalies gravimétriques de la partie sud du craton de Sao Francisco (Brésil). Tectonophysics, 3, 273-293.

https://doi.org/10.1016/0040-1951(81)90101-3

Mbassa, B. J., Kamgang, P., Grégoire, M., Njonfang, E., Benoit, M., Itiga, Z., \& Nfomou, N. (2016). Evidence of Heterogeneous Crustal Origin for the Pan-African Mbengwi Granitoids and the Associated Mafic Intrusions (Northwestern Cameroon, Central Africa). Comptes Comptes Rendus Geosciences, 348, 116-126. https://doi.org/10.1016/j.crte.2015.09.009

Naïmou, S., Ganwa, A. A., Urs, K., Amadou, D. K., \& Ekodeck, G. E. (2014). Petrography and Geochemistry of Precambrian Basement Straddling the Cameroon-Chad Border: The Touboro-Baïbokoum Area. International Journal of Geosciences, 5, 418-431. https://doi.org/10.4236/ijg.2014.54040 
Neves, S. P. (2003). Proterozoic History of the Borborema Province (NE 946 Brazil): Correlations with Neighboring Cratons and Pan-African 947 Belts, and Implications for the Evolution of Western Gondwana. Tectonics, 22 p.

https://doi.org/10.1029/2001TC001352

Njanko, T., Nédélec, A., \& Affaton, P. (2006). Synkinematic High-K Calc-Alkaline Plutons Associated with the Pan-African Central Cameroun Shear Zone (W-Tibati Area): Petrology and Geodynamic Significance. Journal of African Earth Sciences, 44, 494-510.

https://doi.org/10.1016/j.jafrearsci.2005.11.016

Norcross, C., Davis D. W., Spooner E. T. C., \& Rust A., (2000). U-Pb and Pb-Pb Constraints on Paleoproterozoic Magmatism, Deformation and Gold Mineralization in the Omai Area, Guyana Shield. Precambrian Research, 102, 69-86.

https://doi.org/10.1016/S0301-9268(99)00102-3

Nzenti, J. P., Barbey, P., Jegouzo, P., \& Moreau, C. (1984). Un nouvel exemple de ceinture granulitique dans une chaîne Protérozoïque de transition: Les migmatites de Yaoundé au Cameroun. Comptes Rendus de l'Académie des Sciences, Sciences de la Terre et des Planètes, 17, 1197-1199.

Penaye, J., Toteu, S. F., Van Schmus, W. R., \& Nzenti, J. P. (1993). U-Pb and Sm-Nd Preliminary Geochronology Data on the Yaoundé Group, Cameroon: Re-Interpretation of the Granulitic Rock as the Suture of a Collision in the "Centrafrican Belt". Comptes Rendus de l'Académie des Sciences Paris, 317, 789-794.

Pinna, P., Calvez, J.-Y., Abessolo, A., Angel, J.-M., Mekoulou-Mekoulou, T., Mananga, G., \& Vernhet, Y. (1994). Neoproterozoïc Events in the Tcholliré Area: Pan-African Crustal Growth and Geodynamics in Central-Northern Cameroon (Adamawa and North Provinces). Journal of African Earth Sciences, 18, 347-353. https://doi.org/10.1016/0899-5362(94)90074-4

Poidevin, J.-L. (1985). Le Protérozoïque supérieur de la République centrafricaine. Annuaire du Musée Royal d'Afrique Centrale, 91, 75.

Regan, R. D., \& Marsh, B. D. (1982). The Bangui Magnetic Anomaly: Its Geological Origin. Journal of Geophysical Research, 87, 1107-1120. https://doi.org/10.1029/JB087iB02p01107

Renné, P. R., Onstott, T. C., D’Agrella Filho, M. S., Pacca, I. G., \& Teixeira, W. (1990). 40Ar/39Ar Dating of 1.0-1.1 Ga Magnetizations from the São Francisco and Kalahari cratons: Tectonic Implications for Pan-African and Brasiliano Mobile Belts, Earth Planet. Science Letters, 101, 349-366. https://doi.org/10.1016/0012-821X(90)90165-T

Rogers, J. J. W. (1996). A History of Continents in the Past Three Billion Years. Journal of Geology, 104, 91-107. https://doi.org/10.1086/629803

Saha-Fouotsa, A. N. (2018). Croissance et remobilisation crustale au Néoprotérozoïque : magmatisme, métamorphisme et déformation le long de la zone de cisaillement de Tcholliré-Banyo (région de Mbé-Sassa-Mbersi) (384 p). Unpublished Thesis, University of Ngaoundéré.

Saha-Fouotsa, A. N., Tchameni, R., Nomo Negue, E., Daouda, D., Penaye, J., \& Fosso Tchunte, P. M. (2018). Polyphase Deformation in the Mbé-Sassa-Bersi Area: Implications on the Tectono-Magmatic History of the Area and the Tectonic Evolution of the Tcholliré-Banyo and Central Cameroon Shear Zones (Central North Cameroon). Journal of Geosciences and Geomatics, 6, 41-54. https://doi.org/10.12691/jgg-6-2-2

Saha-Fouotsa, A. N., Vanderhaeghe, O., Barbey, P., Eglinger, A., Tchameni, R., Zeh, A., Fosso Tchunte, P., \& Nomo, E. N. (2019). The Geologic Record of the Exhumed Root of the Central African Orogenic Belt in the Central Cameroon Domain (Mbé-Sassa-Mbersi Region). Journal of African Earth Sciences, 151, 286-314. 
https://doi.org/10.1016/j.jafrearsci.2018.12.008

Santos, E. J. (1995). O complexo granítico Lagoa das Pedras: Acresc, ão e colisão na região de Floresta (Pernambuco) Província da Borborema (220 p). Doctor Thesis, Universidade de São Paulo.

Santos, E. J., Van Schmus, W. R., Kozuch, M., \& Brito Neves, B. B. (2010). The Cariris Velhos Tectonic Event in Northeast Brazil. Journal of South American Earth Sciences, 29, 61-76. https://doi.org/10.1016/j.jsames.2009.07.003

Schobbenhaus, C. (1996). As tafrogêneses superpostas Espinhaço e Santo Onofre, Estado da Bahia: Revisão e novas propostas. Revista Brasileira de Geociências, 26, 265-275. https://doi.org/10.25249/0375-7536.19964265276

Silva, A. M., Chemale, Jr. F., Kuyumjian, R. M., \& Heaman, L. (1995). Mafic Dike Swarms of Quadrilátero Ferrífero and Southern Espinhaco, Minas Gerais, Brazil. Revista Brasileira Geociências, 25, 134-137. https://doi.org/10.25249/0375-7536.1995124137

Souza, S. H. P., \& Moura, C. A. V. (1995). Idades Pb-Pb em Zircōes das rochas do Embasamento do Cinturāo Araguaia na Regiāo de Paraiso do Tocantins (TO) (pp. 95-97). Bol. Res. Exp.

Tack, L., Wingate, M. T. D., Liégiois, J.-P., Fernandez-Alonso, M., \& Deblond, A. (2001). Early Neoproterozoic magmatism (1000-910 Ma) of the Zadinian and Mayumbian Groups (Bas-Congo): Onset of Rodinia Rifting at the Western Edge of the Congo Craton. Precambrian Research, 110, 227-306. https://doi.org/10.1016/S0301-9268(01)00192-9

Tchakounté, J., Eglinger, A., Toteu, S. F., Zeh, A., Nkoumbou, C., Mvondo-Ondoa, J., Penaye, P., De Wit, M., \& Barbey, P. (2017). The Adamawa-Yadé Domain, a Piece of Archaean Crust in the Neoproterozoic Central African Orogenic Belt (Bafia Area, Cameroon). Precambrian Research, 299, 210-229. https://doi.org/10.1016/j.precamres.2017.07.001

Tchameni, R., Pouclet, A., Penaye, J., Ganwa, A. A., \& Toteu, S. F. (2006). Petrography and Geochemistry of the Ngaoundéré Pan-African Granitoïds in Central North Cameroon: Implications for Their Sources and Geological Setting. Journal of African Earth Sciences, 44, 511-529. https://doi.org/10.1016/j.jafrearsci.2005.11.017

Teixeira, W., Renne, P. R., Bossi, J., Campai, N., \& D’Agrella Filho, M. S. (1999). 40Ar-39Ar and Rb-Sr Geochronology of the Uruguayan Dike Swarm, Rio de la Plata craton, and Implications for Proterozoic Intraplate Activity in Western Gondwana. Precambrian Research, 93, 153-180. https://doi.org/10.1016/S0301-9268(98)00087-4

Tohver, E., van der Pluijm, B. A., Van der Voo, R., Rizzotto, G., \& Scandolara, J. E. (2002). Paleogeography of the Amazon Craton at 1.2 Ga: Early Greenvillian Collision with the Llano Segment of Laurentia. Earth and Planetary Science Letters, 199, 185-200. https://doi.org/10.1016/S0012-821X(02)00561-7

Toteu, S. F., Van Schmus, W. R., Penaye, J., \& Michard, A. (2001). New U-Pb, and Sm-Nd Data from Nord-Central Cameroun and Its Bearing on the Pre-Pan-African History of Africa. Precambrian Research, 108, 45-73.

https://doi.org/10.1016/S0301-9268(00)00149-2

Toteu, S.F., Penaye, J., \& Poudjom Djomani, Y. (2004). Geodynamic Evolution of the Pan-African Belt in Central Africa with Special Reference to Cameroon. Canadian Journal of Earth Sciences, 41, 73-85. https://doi.org/10.1139/e03-079

Tupinambá, M., Machado, N., Heilbron, M., \& Ragatky, D. (2007). Meso-Neoproterozoic Lithospheric Extensional Events in the São Francisco Craton and Its Surrounding South American and African Metamorphic Belts: A Compilation of U-Pb Ages. Revista Brasileira de Geociências, 37, 87-91. https://doi.org/10.25249/0375-7536.200737S48791 
Uhlein, A., Trompette, R. R., \& Egydio-Silva, M. (1998). Proterozoic Rifting and Closure, SE Border of the São Francisco Craton, Brazil. Journal of South American Earth Sciences, 11, 191-203. https://doi.org/10.1016/S0895-9811(98)00010-8

Van Schmus, W. R., Fetter, A. H., Brito Neves, B. B., \& Williams, I. S. (1999). Ages of Detrital Zircon Populations from Neoproterozoic Supracrustal Units in NE Brazil: Implications for Assembly of West Gondwanaland. Geological Society of America Abstracts with Programs, 31, A-299.

Van Schmus, W. R., Oliveira, E. P., Silva Filho, A. F., Toteu, S. F., Penaye, J., \& Guimarães, I. P. (2008). Proterozoic Links between the Borborema Province, NE Brazil, and the Central African Fold Belt. Geological Society of London. Special Publication, 294, 69-99. https://doi.org/10.1144/SP294.5

Viana, R. R., \& Battilani, G. A. (2014). SHRIMP U-Pb and U-Pb Laser Ablation Geochronological on Zircons from Monte Santo Alkaline Intrusive Suite, Westhern Araguaia Belt, Tocantins State, Brazil. Journal of Geoscience and Environment Protection, 2, 170-180. https://doi.org/10.4236/gep.2014.23022

Vicat, J.-P., \& Pouclet, A. (1995). Nature du magmatisme lié à une extension pré-panafricaine: Les dolérites des bassins de Comba et de Sembé-Ouesso. Bulletin de la Société Géologique de France, 166, 355-364. https://doi.org/10.2113/gssgfbull.166.4.355

Vicat, J.-P., Moloto-A-Kenguemba, G., \& Pouclet, A. (2001). Les granitoïdes de la couverture protérozoïque de la bordure nord du craton du Congo (Sud-Est du Cameroun et Sud-Ouest de la République centrafricaine), témoins d'une activité magmatique post-kibarienne à pré-panafricaine. Earth and Planetary Sciences, 332, 235-242. https://doi.org/10.1016/S1251-8050(01)01521-X

Vicat, J.-P., Pouclet, A., Nkoumbou, C., \& Sémé Mouangué, A. (1997). Le volcanisme fissural néoprotérozoïque des séries du Dja inférieur, de Yokadouma (Cameroun) et de Nola (Centrafrique): Signification géotectonique. Comptes rendus de l'Académie des Sciences, 325, 671-677. https://doi.org/10.1016/S1251-8050(97)89109-4

Zeh, A., \& Gerdes, A. (2012). U-Pb and Hf Isotope Record of Detrital Zircons from Gold-Bearing Sediments of the Pietersburg Greenstone Belt (South Africa): Is There a Common Provenance with the Witwatersrand Basin? Precambrian Research, 204-205, 46-56. https://doi.org/10.1016/j.precamres.2012.02.013 